\title{
Selection of Conserved Epitopes from Hepatitis C Virus for Pan-Populational Stimulation of T-Cell Responses
}

\author{
Magdalena Molero-Abraham, ${ }^{1}$ Esther M. Lafuente, ${ }^{1}$ \\ Darren R. Flower, ${ }^{2}$ and Pedro A. Reche ${ }^{1}$ \\ ${ }^{1}$ Facultad de Medicina, Sección de Inmunología, Universidad Complutense de Madrid, Avenida Complutense S/N, \\ 28040 Madrid, Spain \\ ${ }^{2}$ School of Life and Health Sciences, University of Aston, Aston Triangle, Birmingham B4 7ET, UK
}

Correspondence should be addressed to Pedro A. Reche; parecheg@med.ucm.es

Received 2 August 2013; Accepted 19 September 2013

Academic Editor: Masha Fridkis-Hareli

Copyright (C) 2013 Magdalena Molero-Abraham et al. This is an open access article distributed under the Creative Commons Attribution License, which permits unrestricted use, distribution, and reproduction in any medium, provided the original work is properly cited.

\begin{abstract}
The hepatitis $\mathrm{C}$ virus (HCV) is able to persist as a chronic infection, which can lead to cirrhosis and liver cancer. There is evidence that clearance of HCV is linked to strong responses by CD8 cytotoxic T lymphocytes (CTLs), suggesting that eliciting CTL responses against HCV through an epitope-based vaccine could prove an effective means of immunization. However, HCV genomic plasticity as well as the polymorphisms of HLA I molecules restricting CD8 T-cell responses challenges the selection of epitopes for a widely protective vaccine. Here, we devised an approach to overcome these limitations. From available databases, we first collected a set of $245 \mathrm{HCV}$-specific CD8 T-cell epitopes, all known to be targeted in the course of a natural infection in humans. After a sequence variability analysis, we next identified 17 highly invariant epitopes. Subsequently, we predicted the epitope HLA I binding profiles that determine their potential presentation and recognition. Finally, using the relevant HLA I-genetic frequencies, we identified various epitope subsets encompassing 6 conserved HCV-specific CTL epitopes each predicted to elicit an effective T-cell response in any individual regardless of their HLA I background. We implemented this epitope selection approach for free public use at the EPISOPT web server.
\end{abstract}

\section{Introduction}

Hepatitis $\mathrm{C}$ virus (HCV) causes a chronic infection that produces liver fibrosis (20\% of infected individuals) which can lead to liver failure and the development of liver carcinomas [1]. An estimated 170 million people (3\% of the world population) are HCV carriers. Moreover, the prevalence of the infection and associated complications are increasing [1]. Standard therapy against HCV consists of a combination of interferon and ribavirine. This treatment improves symptoms and survival prognosis of patients infected with HCV. However, $50 \%$ of patients do not respond adequately, with genotype 1 being especially refractory to treatment [2]. Thus, the development of alternative treatments against $\mathrm{HCV}$, including an effective vaccine, is of paramount importance.

No vaccine is currently available for $\mathrm{HCV}$ infection [3]. With the increasing discovery of new anti-HCV small molecule drugs, some may argue that liver disease associated with $\mathrm{HCV}$ will largely disappear and thus that $\mathrm{HCV}$ vaccine discovery will become superfluous. However, few of the 170+ million HCV infected population will have access to costly medications. Nor will these medicines treat all patients with equal effectiveness. Therefore, prophylactic treatment of $\mathrm{HCV}$ infection by a vaccine represents a cost effective and efficient alternative to medicines. Intravenous drug usersthe major group suffering new $\mathrm{HCV}$ infections in most locations-have an increased likelihood of reinfection and 
would thus also benefit from prophylactic treatments able to prevent chronic $\mathrm{HCV}$ infection.

HCV exhibits extraordinary genetic diversity, including at least 6 genotypes $(1(\mathrm{a}, \mathrm{b}, \mathrm{c}), 2(\mathrm{a}, \mathrm{b}, \mathrm{c}), 3(\mathrm{a}, \mathrm{b}), 4 \mathrm{a}, 5 \mathrm{a}$, and $6 a)$ and 52 different subtypes [2, 4], and this represents a major challenge for vaccine development. Work in humans and chimpanzees (the only animal model susceptible to $\mathrm{HCV}$ ) shows that antibodies are not decisive in resolution and control of HCV infection $[5,6]$ possibly because this response is focused on regions of the virus envelope, which experiences extreme sequence variability. In contrast, there is evidence that $\mathrm{T}$ cells play a fundamental role in the control of $\mathrm{HCV}$ infection, since viral clearance observed during acute transient infection correlates with the activation of CD4 T helper (Th) lymphocytes and, importantly, with a broad and sustained response by cytotoxic CD8 T lymphocytes (CTLs) $[7,8]$. In contrast, these responses are weak and limited in patients with chronic infection [5, 9-13]. CTLs contribute to the containment of viral infection by eliminating viralinfected cells and indirectly by secretion of antiviral factors [14], while CD4 Th cells cooperate to produce cytokines that stimulate effector functions of CTLs [15]. Together, this suggests that the development of a vaccine against $\mathrm{HCV}$ will likely rely on the induction of $\mathrm{T}$-cell responses and in particular a CTL response. It has been shown that it is possible to induce potent and specific CTL responses when immunizing with adenoviral vectors encoding $\mathrm{HCV}$ products [16] and to protect chimpanzees from HCV infection [17]. However, given the extreme mutability of $\mathrm{HCV}$, it is difficult to conceive that acquired immunity to a single HCV strain could protect against other, clearly divergent, strains [18] or prevent the emergence of mutants that escape immune responses $[19,20]$.

CTL responses are directed against short peptides, epitopes, presented by human leukocyte antigens (HLA I) expressed on the cell surface of antigen presenting and target cells. Therefore, HCV immune evasion could be avoided by appropriate selection of conserved $\mathrm{HCV}$-specific CTL epitopes. Currently, there are hundreds of bona fide HCVspecific CTL epitopes deposited in specialized databases. However, HCV diversity and extreme polymorphism of HLA I molecules restricting the CTL responses make optimal epitope selection anything but straightforward, particularly if our aim is developing widely protective epitope-based vaccine. For such a task, we started with a set of $245 \mathrm{HCV}$-specific CTL epitopes, known to be targeted in the course of a natural infection, and sought minimal subsets of invariant epitopes that could be basis for a widely protective epitope-based vaccine. We found various epitope subsets encompassing 6 conserved HCV-specific CTL epitopes that we predicted could elicit an effective T-cell response in any individual regardless of their HLA I background. Here, we analyzed these results and discussed the reason for epitope conservation. We also introduced EPISOPT (http://imed.med.ucm.es/Tools/episopt.html), a server that implements our approach for selecting epitope combinations providing the largest population protection coverage.
TABLE 1: HCV genotype of polyproteins used in this study.

\begin{tabular}{lc}
\hline Genotype & Sequences \\
\hline 1 & 501 \\
2 & 50 \\
3 & 7 \\
4 & 34 \\
5 & 2 \\
6 & 61 \\
Unassigned $^{*}$ & 29 \\
\hline
\end{tabular}

${ }^{*}$ Sequences could not be readily classified onto any of the 6 major genotypes. Genotypes were assigned using MuLDAS (http://www.muldas .org/MuLDAS/).

\section{Materials and Methods}

2.1. HCV-Specific CTL Epitopes. We used the EPIMHC [21] and IEDB [22] databases to identify HCV-specific CTL epitopes. Specifically, we collected a set of 245 peptide sequences comprising HCV-specific CTL epitopes. These peptides were reported to stimulate the response of human CTLs elicited in the course of a natural infection by HCV in humans and all have nine residues. When available, we also collected the human MHCI molecule that was determined to restrict the CTL response against the peptide. This peptide set is provided as Supplementary Material in Additional File S1 available online at http://dx.doi.org/10.1155/2013/601943 and will be also provided by the corresponding author upon writing request.

2.2. Retrieval, Processing, and Multiple Sequence Alignment of HCV Polyproteins. We generated a multiple sequence alignment (MSA) encompassing 684 entire HCV-polyproteins using MUSCLE [23] with default settings. The HCVpolyproteins included representatives from all HCV genotypes (Table 1). We obtained them from the translation feature (CDS) of $\mathrm{HCV}$ nucleotide records retrieved after GenBank genomic accessions identified in the European HCV database (EuHCVdb). To ensure working with fulllength polyproteins, we only considered translated CDS with more than 3000 amino acid residues. The multiple sequence alignment (MSA) is provided as supplementary material in Additional File S1.

2.3. Generation of an HCV-Reference Sequence with the Variables Sites Masked. We generated a HCV-reference sequence by calculating the sequence variability in the MSA of HCVpolyproteins using the Shannon entropy [24], $H$, as a variability metric [25-27]. Briefly, the Shannon entropy is given by

$$
H=-\sum_{i=1}^{M} P_{i} \log _{2} P_{i},
$$

where $P_{i}$ is the fraction of residues of amino acid type $i$ and $M$ is equal to 20 , the number of amino acid types. $H$ 
ranges from 0 (total conservation, only one amino acid type is present at that position) to 4.322 (all 20 amino acids are equally represented in that position).

We assigned the sequence variability, $H$, to the HCVpolyprotein obtained from GenBank accession M62321 (protein id: AAA45676.1) and subsequently masked all positions with a variability, $H$, greater than 0.5 , ensuring that the remaining residue positions are highly conserved $[28,29]$.

\subsection{Prediction of Epitope Presentation by HLA I and Computa-} tion of Population Protection Coverage (PPC). CTL epitopes are peptides presented by HLA I molecules and recognized by cytotoxic CD8 T lymphocytes (CTLs). For HLA I molecules can only present to $\mathrm{T}$ cells those peptides that bind to them, we anticipated HLA I presentation of epitomes/peptides by predicting HLA I binding. Specifically, we used 55 HLA I-specific motif position-specific scoring matrices (PSSMs) [30-32] to predict epitope binding to the corresponding HLA I molecules. We considered that a given peptide binds to specific HLA I molecule when its binding score ranks within the top $3 \%$ percentile of the binding scores computed for 1000 random 9mer peptides (average amino acid composition of proteins in the SwissProt database).

The population protection coverage (PPC) of an arbitrary set of CTL epitopes is given by the proportion of the population that could potentially mount an immune response to any of these epitopes and can be computed by knowing the gene frequencies of the HLA I alleles that can present the epitopes. Given the required HLA I restriction of CTL responses, the PPC for a set of CTL epitopes matches the proportion of the population that exhibits at least one of the HLA I alleles that can bind and present any of those epitopes, which corresponds to the cumulative phenotype frequency (CPF) of the targeted HLA I alleles. In this work, we computed $\mathrm{CPF}$ using the equations described elsewhere in [26] and the HLA I allelic and haplotype frequencies published by Cao et al. [33] for 5 major American ethnic groups (Black, Caucasian, Hispanic, Native American, and Asian). HLA I allele frequencies published by Cao et al. [33] cover most common HLA-A, -B, and -C alleles; 10-20\% of all available HLA I sequences in IMGT/HLA database [34].

2.5. Identification of Optimal Epitope Combinations with a Given Population Protection Coverage. In order to identify minimal sets of epitopes (optimal epitope combinations) with a target PPC within a starting set of CD8 T-cell epitopes, we first find the HLA I molecules that could present those epitopes by predicting their binding to 55 HLA I molecules (HLA I binding profile), as indicated elsewhere in Section 2. With the predicted HLA I binding profiles, we can compute CPF and, hence, epitope PPC. Once we have the epitope HLA I binding profiles, we search for epitope combinations from the initial set of epitopes, starting with one epitope and iteratively increasing the number of epitopes being considering, which reach the target PPC. At each iteration, we also computed the largest PPC that can be reached with the number of epitopes being considered. Because PPC vary for the 5 ethnic groups for which we have HLA I gene allele frequencies, we seek that the target PPC is reached in each ethnic group. We made this method available for identifying minimal set of epitope combinations reaching a target PPC through the EPISOPT web server. EPISOPT is implemented on an Apache Web server running under the Mac OSX operating system. The EPISOPT functional core consists of a PERL CGI (Common Gateway Interface) script that handles the input, executes the above outlined methods, and then assembles and displays the results.

2.6. Other Procedures. We identified three-dimensional (3D) structures of HCV proteins upon BLAST searches [35] with the epitope sequences against the $\mathrm{PDB}$ sequence database at NCBI (http://blast.ncbi.nlm.nih.gov/Blast.cgi). We obtained solvent accessibility from the relevant 3D structures using the program NACCESS [36], and we used the server MAST (http://imed.med.ucm.es/Tools/msat.html) for mapping the accessibility onto the $3 \mathrm{D}$ structures.

\section{Result and Discussion}

HCV vaccines could prevent chronic HCV infection, a leading cause of both liver fibrosis and liver cancer. Unfortunately, unlike hepatitis A virus (HAV) and hepatitis B virus (HBV) $[37,38]$, no such $\mathrm{HCV}$ vaccine exists. Most vaccines pursue the generation of long lasting immunity mediated by neutralizing antibodies. Thus, current vaccines for HAV and HBV are based on viral-specific recombinant proteins eliciting such type of immunity $[38,39]$. In contrast, immunization trials with recombinant $\mathrm{HCV}$ proteins have all failed [37], likely because antibodies do not play a key role in the control of the infection by $\operatorname{HCV}[5,6]$. Targeting the cellular arm of the adaptive immune system residing in the $\mathrm{T}$ cells is surely the key for the development of a successful HCV vaccine $[7,8]$, and multiple Tcell epitope-based vaccines are particularly suitable for that task.

HCV diversity and HLA polymorphisms are a handicap for developing a broadly protective T-cell epitope-based vaccine against $\mathrm{HCV}$. Therefore, epitope selection is a key step for epitope-vaccine design. Given the relevance of CTL responses in the containment of HCV acute infection [7, 8], in this work we sought to find invariant $\mathrm{HCV}$-specific CTL epitopes that could serve to develop approach depicted in Figure 1.

A key feature of the approach is the use of already discovered CTL epitopes. Previous work has yielded large numbers of HCV-specific epitopes, which are available in specialized databases such SYFPEITHI [40], JenPep [41], and MHCBN [42], TEPIDAS [43], Immune Epitope database [44], and EPIMHC [21]. In our study, we collected the HCVspecific epitopes from the EPIMHC and Immune Epitope databases. We sought published, annotated epitopes that are targeted in the course of a natural HCV infection and 


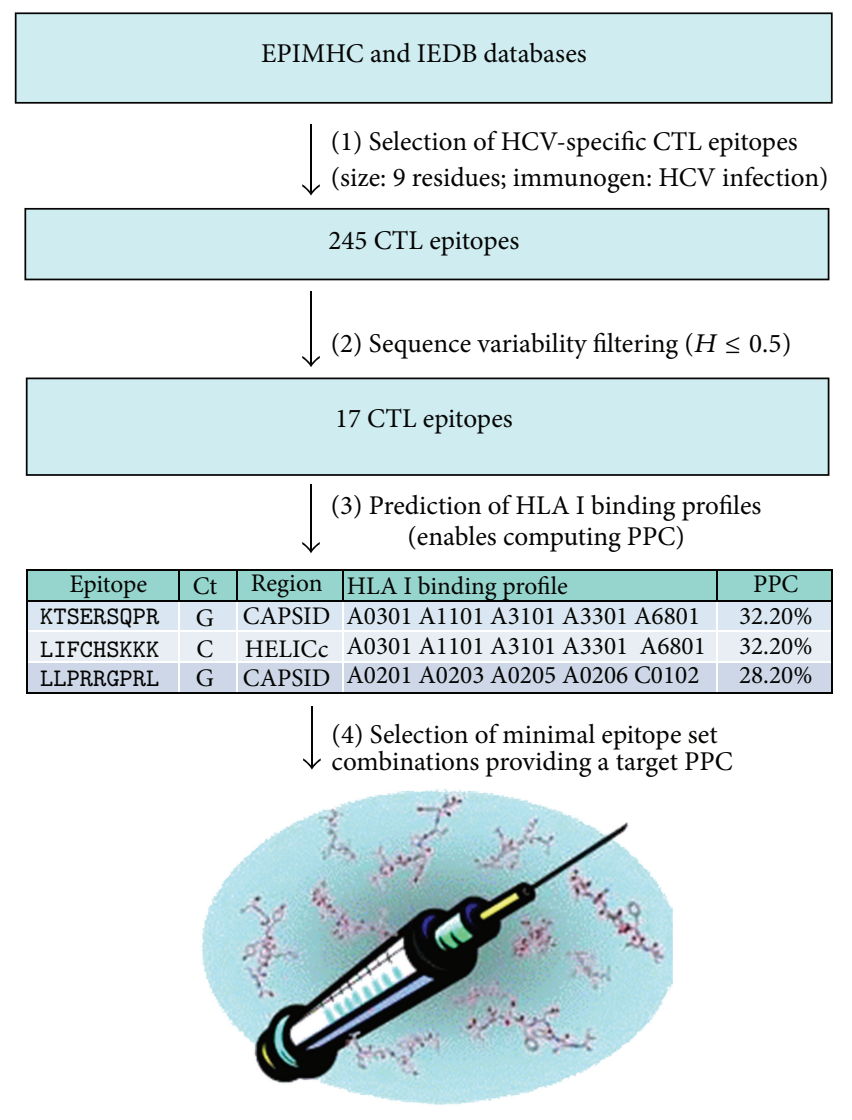

FIGURE 1: HCV epitope selection approach. Overview of the approach devised to select invariant $\mathrm{HCV}$-specific CTL epitopes that could be the basis for a broadly protective epitope-based vaccine against HCV. The approach consists of 4 basic steps: (1) selection of epitopes from databases according to the indicated criteria. Immunogen HCV infection means that all selected HCV epitopes were reported to be targeted in the course of a natural infection in humans. They are immunogenic. (2) Sequence variability filtering. We only considered epitopes that do not contain any residue with a variability given by Shannon entropy, $H$, greater than 0.5 . (3) Prediction of epitope HLA I binding profiles which enables computation of the percentage of the population that will be able to elicit a T-cell response against a particular epitope (PPC). (4) Selection of subsets of epitopes that with a minimum number of epitopes reach a target PPC.

a size of nine residues, assembling a set of $245 \mathrm{HCV}$ specific CTL epitopes (provided as supplementary material in Additional File S1). The bases for the selection criteria are the following. Appropriate antigen processing is a prerequisite for HLA presentation and limits T-cell recognition $[45,46]$. By considering CTL epitopes that are targeted in the course of a natural $\mathrm{HCV}$ infection, we assumed that (A) antigen processing is conserved (it will be futile to elicit a T-cell response against an epitope that it is not processed and available for presentation in the course of an infection with $\mathrm{HCV}$ ) and (B) epitope presentation and T-cell recognition is only determined by HLA I binding. As for the size of the epitopes, HLA I molecules bind and present peptides from 8 to 11 residues but most of them have 9 residues [30]. There are reports in the literature echoed in the databases of much large peptides, up 30 amino acids, that are capable of eliciting CD8 T-cell responses. These peptides do not represent optimal CD8 T-cell epitopes, as they need further processing prior to HLA I binding.

The next step in our approach (Figure 1) was the identification of highly conserved epitopes. In order to do so, we first carried out a variability analysis of a multiple sequence alignment (MSA) of HCV-polyproteins using Shannon entropy, $H$ (details in Section 2). The MSA included HCV polyproteins from all 6 genotypes; however, genotype 1 was the most represented, with 501 sequences (Table 1). Subsequently, we masked in an HCV-polyprotein reference sequence (AN: AAA45676.1) all variable sites within the MSA with $H>0.5$. We mapped variability onto this sequence rather than the artificial conserved sequence for two reasons: first, conserved sequences derived upon a MSA tend to be too long and contain stretches of amino acids not present in any real sequence, and secondly, AAA45676.1 has long been used as a reference sequence to design overlapping peptides and check T-cell responses. HCV-polyprotein AAA45676.1 with the variable sites masked is depicted in Figure 2 showing the location of the mature proteins. We used this sequence to discard those epitopes that have any residue, including those flanking the C-terminus, with $H>0.5$. The residue flanking the C-terminus of the CTL epitope is a determinant for proteasome cleavage, and mutations in that residue can abrogate T-cell recognition [47]. Thus, we also required that residue to be invariant. Only 17 epitopes meet these criteria and are shown in Table 2. That such a few peptides have all their residues with entropy $H \leq 0.5$ reflects the large genomic plasticity of HCV.

In general, most of the invariant epitopes reside in the CAPSID and CORE proteins of HCV. One reason for this preferred location is that these two proteins seem to be the most conserved. However, regardless of such conservation, we recently showed that proteins located at the $\mathrm{N}$ terminus of polyproteins bear more epitopes than expected for the size because they are preferentially translated [25]. To find structure-function determinants for the conservation of these particular 17 epitopes, we identified three-dimensional structures of HCV proteins bearing the identified epitopes (Table 2) and mapped them onto the 3D structure (Figure 3) (see details in Section 2). We could only map 5 of the 17 conserved epitopes: four of them, LIFCHSKKK, HSKKKCDEL, ITYSTYGKF, and TYSTYGKFL mapped onto two different domains of the NS3 helicase (Figures 3(a) and 3(b)), while one, TIMAKNEVF, mapped onto the RNA polymerase of HCV (Figure 3(c)). Note that the epitopes LIFCHSKKK and HSKKKCDEL overlap and so the epitopes ITYSTYGKF and TYSTYGKFL. Interestingly, all these epitopes bear a large proportion of residues that are hydrophobic and buried in the 3D structure (shown in blue in Figures 3(a), 3(b), and $3(\mathrm{c})$ ). The relative accessibility of each of the epitope residues is shown in Figure 4. For example, the fragment ITYSTYGKFL (it contains two overlapping epitopes) and 


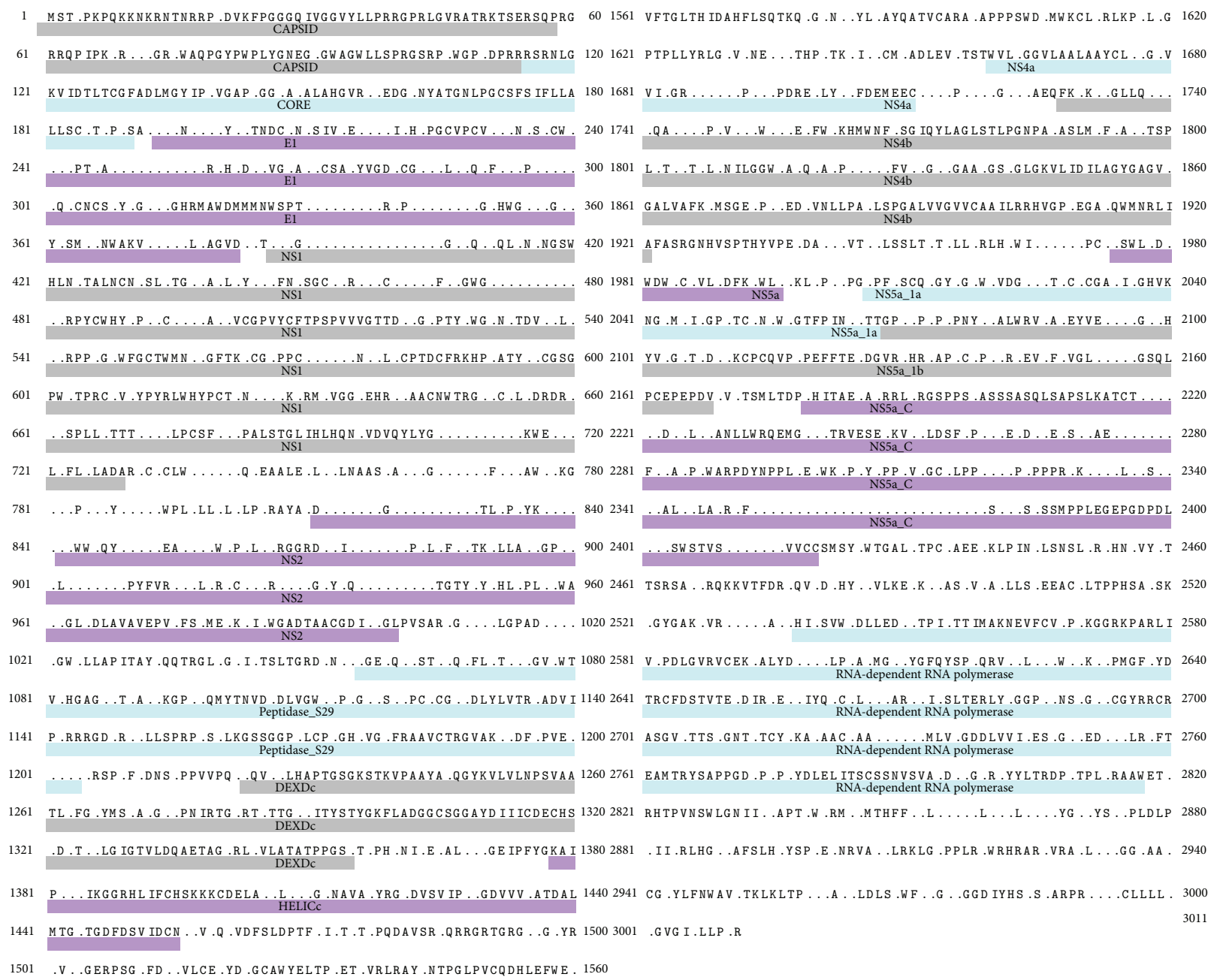

FIGURE 2: HCV polyprotein with variable positions masked. The figure shows the amino acid sequence HCV-polyprotein (protein id; AAA45676.1) with residues positions with a sequence variability $>0.5$ masked (shown as dots). We computed sequence variability from a multiple sequence alignment of HCV polyproteins using Shannon entropy, $H$, and masked the variable sites on the reference sequence as detailed in Section 2. The mature proteins that arise upon HCV-polyprotein processing are indicated.

the epitope TIMAKNEVF only contain residues that are completely buried or semiburied and many are hydrophobic (Figures 3 and 4). These residues form part of the protein hydrophobic core and are key for protein stability. Therefore, the selected epitopes are conserved as they comprise residues involved in protein stability.

Because we aimed to set the basis for a broadly protective epitope-based vaccine against HCV, our next move was to be able to compute the expected population protection coverage (PPC) of any given set of epitopes, defined as the percentage of the population that would be able to elicit a T-cell response against any of them. To this end, we first predicted the binding of each conserved epitope to 55 HLA I molecules for which we have suitable predictors (details in Section 2) thus obtaining predicted epitope HLA I binding profiles. We assumed that any epitope that binds to a given HLA I molecule would be presented and able to elicit a T-cell response. Under this scenario, the HLA I binding profile of a given epitope determines its potential PPC, and we computed it using genetic HLA I frequencies for 5 ethnic groups in the American population [33] (see Section 2 for a detailed explanation). In Table 2, we show the predicted HLA I binding profiles of the 17 conserved HCV-specific epitopes. With this data, we then aimed to identify epitope combinations reaching a 95\% PPC in all five ethnic groups being considered, using the method, described in Section 2. This method does not guarantee any multispecificity, as it only seeks for epitope combinations reaching a target PPC. Multispecificity is a common scenario in the course of natural infection as any given individual will elicit $\mathrm{T}$-cell responses against at least two or three immunodominant pathogen-specific epitopes [49].

We found that a PPC > $80 \%$ can be reached with just 3 epitopes, LLPRRGPRL, KTSERSQPR/LIFCHSKKK (both 
TABLE 2: Selected conserved HCV-specific T-cell epitopes with predicted HLA I binding profiles.

\begin{tabular}{|c|c|c|c|c|c|c|c|}
\hline Epitope & $\mathrm{Ct}$ & Region & $\mathrm{N}$ & $3 \mathrm{D}$ & $3 \mathrm{D}-\mathrm{N}$ & PPC & HLA I binding profile \\
\hline KTSERSQPR & G & CAPSID & 71 & NA & - & $32.2 \%$ & A0301 A1101 A3101 A3301 A6801 \\
\hline LIFCHSKKK & $\mathrm{C}$ & HELICc & 1391 & 2F55|A & $365-373$ & $32.2 \%$ & A0301 A1101 A3101 A3301 A6801 \\
\hline LLPRRGPRL & G & CAPSID & 36 & 1XCQ|P & - & $28.2 \%$ & A0201 A0203 A0205 A0206 C0102 \\
\hline LPGCSFSIF & $\mathrm{L}$ & CORE & 169 & NA & - & $27.8 \%$ & B0702 B1502 B1508 B3501 B5301 B5401 \\
\hline DPRRRSRNL & G & CAPSID/CORE & 101 & NA & - & $26.9 \%$ & B0702 B0801 B5101 B5102 B5103 B5301 B5401 \\
\hline GFADLMGYI & $\mathrm{P}$ & CORE & 129 & NA & - & $6.3 \%$ & A0202 A0203 A0205 A6802 B3801 \\
\hline NLPGCSFSI & $\mathrm{F}$ & CORE & 168 & NA & - & $5.9 \%$ & A0205 C0102 \\
\hline SFSIFLLAL & $\mathrm{L}$ & CORE & 173 & NA & - & $5.5 \%$ & A2402 \\
\hline TYSTYGKFL & A & DEXDC & 1292 & 2F55|A & $266-274$ & $5.5 \%$ & A2402 \\
\hline ITYSTYGKF & $\mathrm{L}$ & DEXDC & 1291 & 2F55|A & $265-273$ & $2.7 \%$ & B1516 B1517 B5702 B5801 \\
\hline HSKKKCDEL & A & HELICc & 1395 & 2F55|A & $369-377$ & $2.5 \%$ & B0801 \\
\hline YLLPRRGPR & $\mathrm{L}$ & CAPSID & 35 & 1XCQ|P & - & $1.4 \%$ & A3301 A6601 A6801 \\
\hline GQIVGGVYL & $\mathrm{L}$ & CAPSID & 28 & $1 \mathrm{XCQ} \mid \mathrm{P}$ & - & $0.4 \%$ & A0206 A0214 B1510 B4002 \\
\hline TIMAKNEVF & $\mathrm{C}$ & RNADP & 2587 & 2BRK|A & $137-145$ & 0 & B1513 B5702 \\
\hline GPRLGVRAT & $\mathrm{R}$ & CAPSID & 61 & NA & - & 0 & \\
\hline RLGVRATRK & $\mathrm{T}$ & CAPSID & 63 & NA & - & 0 & \\
\hline STGLIHLHQ & $\mathrm{N}$ & NS1 & 686 & NA & - & 0 & \\
\hline
\end{tabular}

Epitope: amino acid sequence of the epitope; Ct: residue that flanks the C-terminal end of the epitope; Region: protein/domain in HCV polyprotein bearing the epitope; N: position in HCV polyprotein of the epitope N-terminal residue; 3D: PDB code with 3D structure of protein encompassing the epitope; 3D-N: location of the epitope in the 3D structure; PPC: population protection coverage; HLA I binding profile: HLA I molecules predicted to present the epitope. All epitope residues and c-terminal flanking residues have $H \leq 0.5$. PPC values equal to the lower value computed with the gene frequencies corresponding to 5 ethnic populations [33]. PDB 1XCQ|P corresponds to a peptide from HCV capsid bound to an antibody Fab; hence we do not show the location of the epitope in the 3D structure. NA: PDB not available.

epitopes have the same HLA I binding profile), and LPGCSFSIF (Figure 5(a)). This is not surprising as these epitopes seem to target 3 mayor supertypes: A2, A3, and B7 [50-52]. Note, however, that we predicted peptide binding to $\mathrm{MHC}$ I molecules beyond predefined HLA I supertypes. To reach a PPC $\geq 95 \%$ however, we found that 6 epitopes were required. Moreover, we found that there were 15 different 6-epitope combinations, differing in at least one epitope, providing a $\mathrm{PPC} \geq 95 \%$. The largest $\mathrm{PPC}$ reached by one of these epitope combinations was of $97 \%$ and included the following peptide sequences: LLPRRGPRL, GFADLMGY, KTSERSQPR/LIFCHSKKK, SFSIFLLAL, LPGCSFSIF, and ITYSTYGKF. All 6-epitope combinations reaching PPC $\geq$ 95\% are provided as supplementary data in Additional File S3. All these epitopes combinations differ one from each other in at least one epitope. We also analyzed what epitopes were actually included in the resulting solutions. Of all the epitopes in Table 2, only the first 12 were included in at least one of the epitope combinations reaching PPC $\geq 95 \%$, while the last 5 were never included. The frequency at which the peptides were included in these combinations is shown in Figure 5(b) and varied from 15 times for peptide LLPRRGPRL (it was included in each of the epitope combinations reaching $\mathrm{PPC} \geq$ 95\%) to only once for GQIVGGVYL.
Overall, the results shown here indicate that only a handful of conserved HCV-specific epitopes would be needed to elicit CD8 T-cell responses in any individual regardless of their genetic background. We have preliminary data corroborating these predictions. Thus, we have consistently detected responses on $\mathrm{T}$ cells from naïve people primed and expanded using dendritic cells loaded with HCV-peptide pools representing single 6-epitope combinations of PPC $\geq$ $95 \%$ (data not shown). However, we only analyzed the responses in a few individuals and more experiments are underway. It is also important to highlight that our results only provide conserved CTL epitope solutions for a potential epitope-based vaccine against HCV to be broadly protective. We know that the eventual development of an epitopebased vaccine against $\mathrm{HCV}$ is a complex task that will require optimizing peptide-epitope assembling and vaccine delivery [53]. Moreover, such vaccine will also need to incorporate Th epitopes as well as signals/adjuvants for alerting the innate immune system and initiate an adaptive response.

While in this study we focused on HCV, the same approach could easily be extended to other pathogens; there are thousands of CTL epitopes readily available for many other pathogens. To facilitate that end, we introduced 


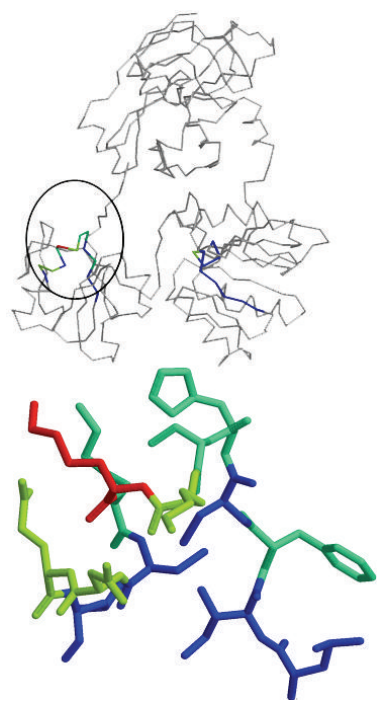

LIFCHSKKKCDEL

(a)

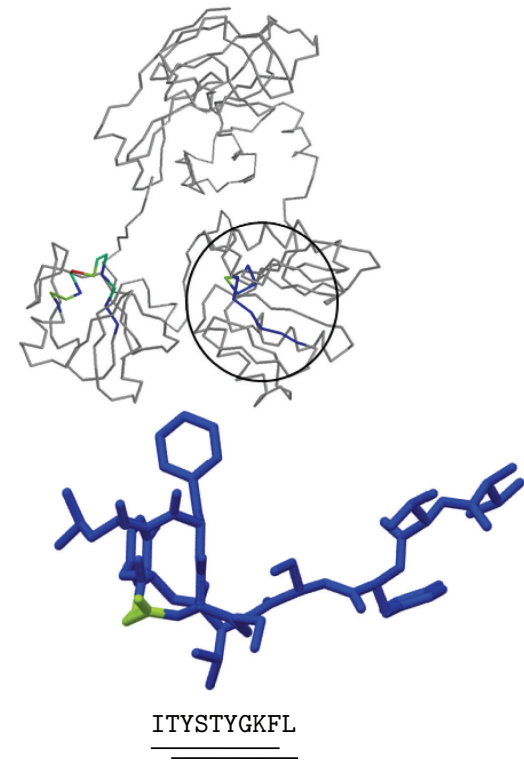

(b)
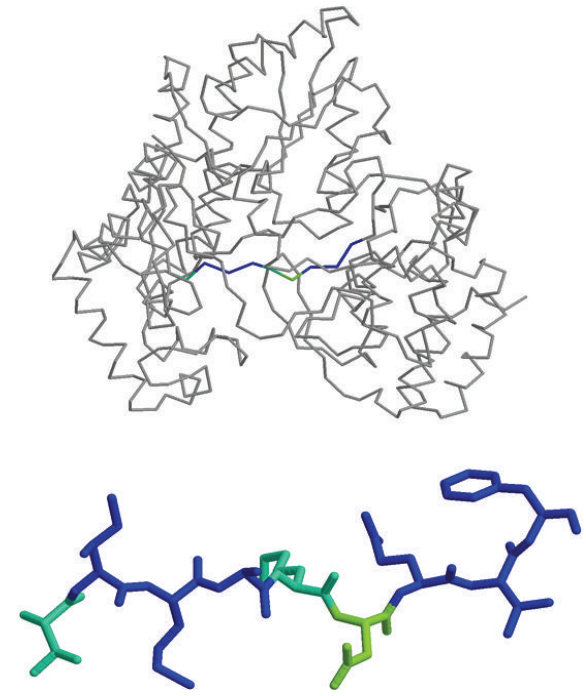

TIMAKNEVF

(c)

FIGURE 3: Three-dimensional structure mapping of HCV-specific invariant CTL epitopes. HCV fragments LIFCHSKKKCDEL and ITYSTYGKFL map onto two different regions of the 3D structure of HCV NS3 helicase (PDB: 2F55), (a) and (b), respectively. Each of these two fragments encompasses two overlapping HCV-specific epitopes. The HCV epitope TIMAKNEVF maps onto HCV polymerase (PDB: 2BRK) (c). Residue color used in the illustrations is related to their solvent accessibility and goes from blue for buried residues to red for accessible residues. Overlapping HCV-specific CTL epitopes are shown underlined. Solvent accessibility was calculated and mapped onto the 3D structures as indicated in Section 2. The figures were rendered using Rasmol [48].

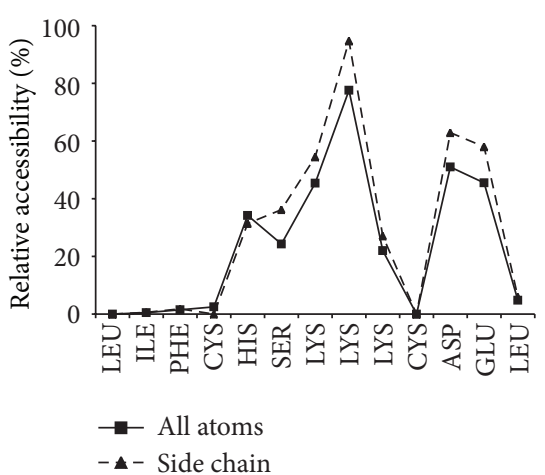

(a)

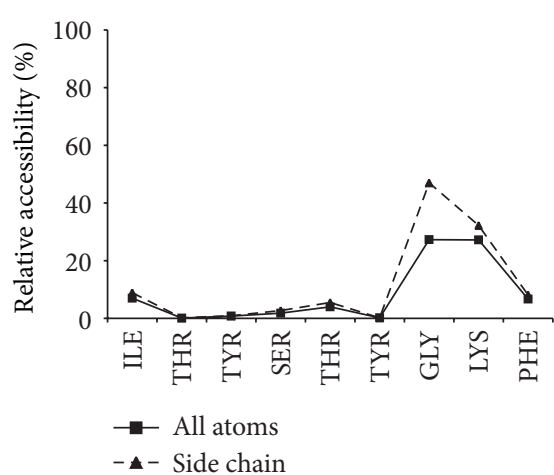

(b)

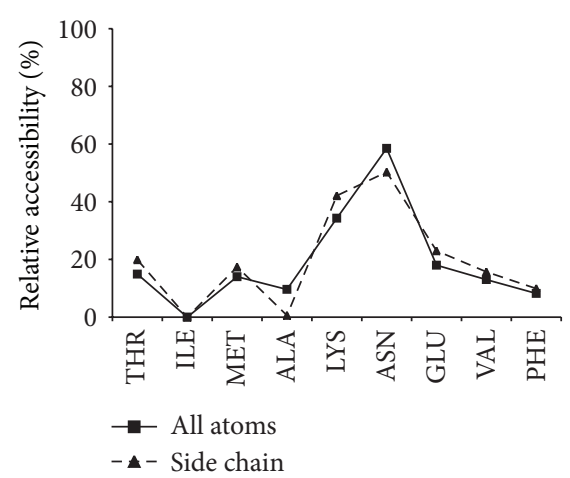

(c)

FIGURE 4: Solvent accessibility of HCV-specific epitopes with counterpart protein 3D structures. The figure depicts a plot of the relative solvent accessibility (\%) ( $y$-axis) of each of the residues ( $x$-axis) in the HCV fragments LIFCHSKKKCDEL (a), ITYSTYGKFL (b) and TIMAKNEVF (c). We plotted both solvent accessibility considering all atoms (squares) and just residue side chains (triangles). Solvent accessibility was calculated from the relevant protein 3D structures using NACCESS [36].

the EPISOPT server (http://imed.med.ucm.es/Tools/episopt .html). The EPISOPT server (Figure 6) implements the steps in our approach consisting of predicting epitope HLA I binding profiles and identifying minimal sets of epitopes reaching a determined PPC. In the future, we will enhance
EPISOPT with sequence variability analyses to select invariant epitopes. EPISOPT is somewhat similar to another online tool developed by Bui et al. [54] available at http:// tools.immuneepitope.org/tools/population/iedb_input. Both tools implement a similar method to compute PPC. However, 


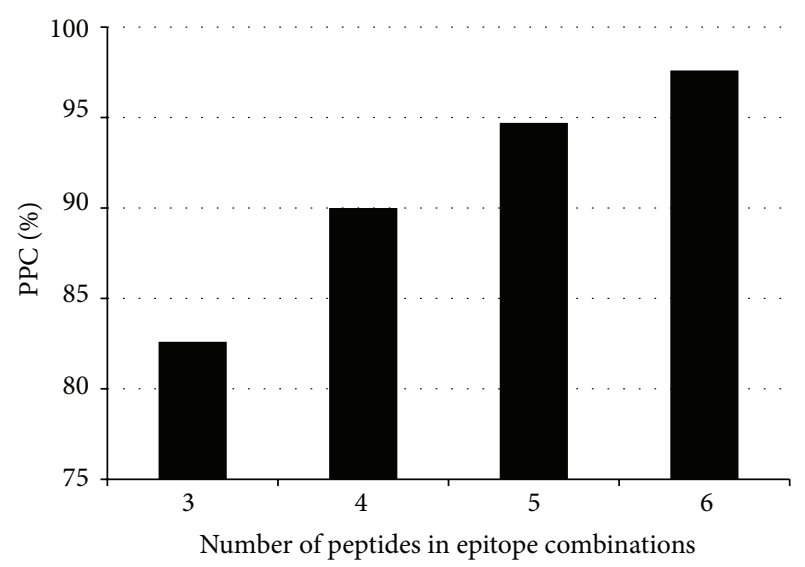

(a)

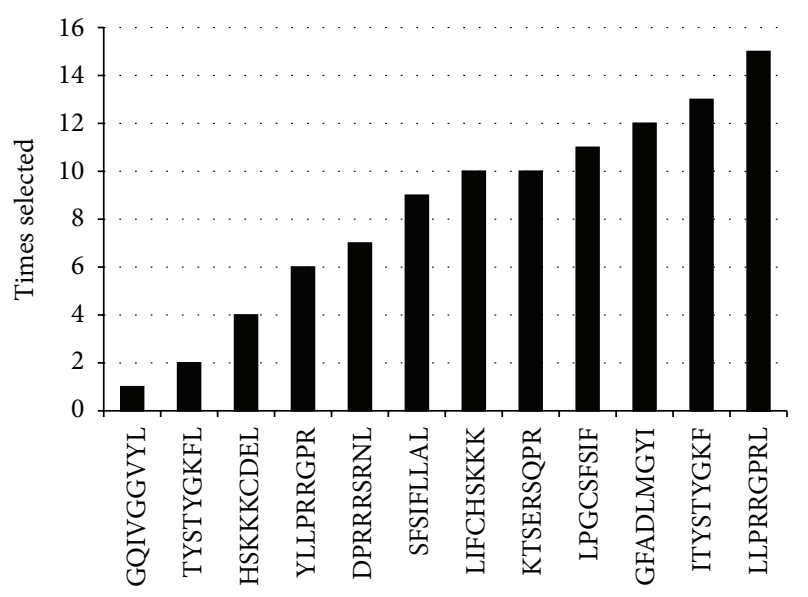

Epitope sequence

(b)

Figure 5: Analysis of HCV-specific CTL epitope set selection. (a) The figure depicts the largest PCC that was reached by HCVspecific CTL epitope combinations containing 3, 4, 5, and 6 epitopes (optimal combinations). As shown, with just 3 epitopes it is possible to reach a $\mathrm{PPC} \geq 80 \%$ and 6 epitopes were required to reach a $\mathrm{PPC} \geq 95 \%$. (b) We found 15 distinct combinations of 6 epitopes each reaching a $\mathrm{PPC} \geq 95 \%$ within the 17 invariant $\mathrm{HCV}$-specific CTL epitopes. In the figure, we depicted the sequence of peptides $(x$-axis) that were included in the mentioned epitope combinations and the times that were included ( $y$-axis).

unlike EPISOPT, Bui et al. [54] tool requires entering the HLA I binding profiles of epitopes as input-it does not predict them-and it does not find epitope combinations reaching a given PPC. We are hopeful that in time, the results and methods derived from this work will make a significant contribution to the design of epitope-based vaccines.

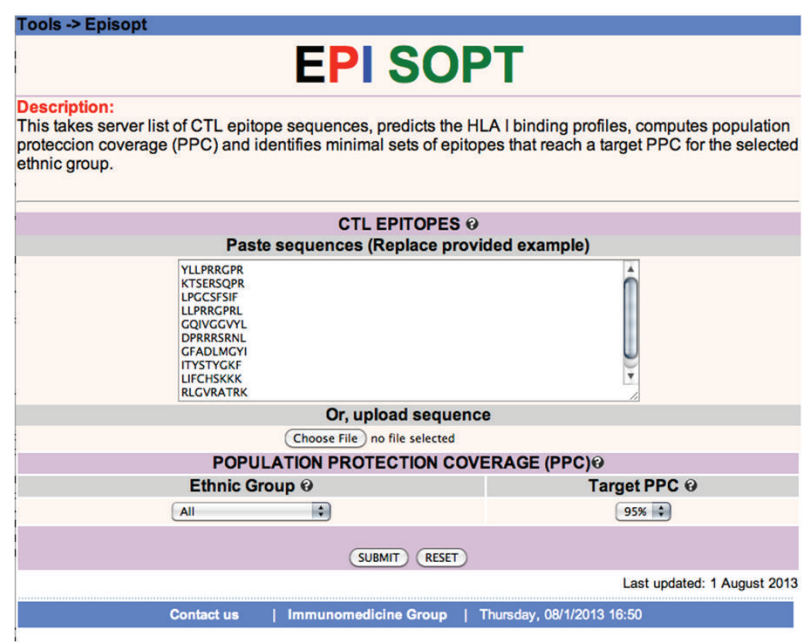

FIGURE 6: EPISOPT web page. The figure shows the input page of EPISOPT. Currently, users enter a list of CTL epitope sequences and select a target PPC and population group. On submission, EPISOPT will find minimal epitope combinations reaching the desired PPC in the selected population group.

\section{Conflict of Interests}

The authors declare that they have no conflict of interests.

\section{Acknowledgment}

This work was supported by Grant SAF2009:08103 from MICCIN to Pedro A. Reche.

\section{References}

[1] T. Poynard, M. Yuen, V. Ratziu, and C. Lung Lai, "Viral hepatitis C," The Lancet, vol. 362, no. 9401, pp. 2095-2100, 2003.

[2] P. Simmonds, J. Bukh, C. Combet et al., "Consensus proposals for a unified system of nomenclature of hepatitis $C$ virus genotypes," Hepatology, vol. 42, no. 4, pp. 962-973, 2005.

[3] M. Houghton and S. Abrignani, "Prospects for a vaccine against the hepatitis C virus," Nature, vol. 436, no. 7053, pp. 961-966, 2005.

[4] J. J. Wilson, S. J. Polyak, T. D. Day, and D. R. Gretch, "Characterization of simple and complex hepatitis $\mathrm{C}$ virus quasispecies by heteroduplex gel shift analysis: correlation with nucleotide sequencing," Journal of General Virology, vol. 76, no. 7, pp. 17631771, 1995.

[5] F. Lechner, D. K. H. Wong, P. R. Dunbar et al., "Analysis of successful immune responses in persons infected with hepatitis C virus," Journal of Experimental Medicine, vol. 191, no. 9, pp. 1499-1512, 2000.

[6] G. Missale, R. Bertoni, V. Lamonaca et al., "Different clinical behaviors of acute hepatitis $\mathrm{C}$ virus infection are associated with different vigor of the anti-viral cell-mediated immune response," Journal of Clinical Investigation, vol. 98, no. 3, pp. 706-714, 1996.

[7] R. Thimme, J. Bukh, H. C. Spangenberg et al., "Viral and immunological determinants of hepatitis $\mathrm{C}$ virus clearance, persistence, and disease," Proceedings of the National Academy 
of Sciences of the United States of America, vol. 99, no. 24, pp. 15661-15668, 2002.

[8] R. Thimme, D. Oldach, K. Chang, C. Steiger, S. C. Ray, and F. V. Chisari, "Determinants of viral clearance and persistence during acute hepatitis C virus infection," Journal of Experimental Medicine, vol. 194, no. 10, pp. 1395-1406, 2001.

[9] P. Sarobe, J. J. Lasarte, N. García, M. P. Civeira, F. BorrásCuesta, and J. Prieto, "Characterization of T-cell responses against immunodominant epitopes from hepatitis $\mathrm{C}$ virus $\mathrm{E} 2$ and NS4a proteins," Journal of Viral Hepatitis, vol. 13, no. 1, pp. 47-55, 2006.

[10] D. G. Bowen and C. M. Walker, "Adaptive immune responses in acute and chronic hepatitis C virus infection," Nature, vol. 436, no. 7053, pp. 946-952, 2005.

[11] K. Deterding, S. V. Pothakamuri, V. Schlaphoff et al., "Clearance of chronic HCV infection during acute delta hepatitis," Infection, vol. 37, no. 2, pp. 159-162, 2009.

[12] S. Gaudieri, A. Rauch, L. P. Park et al., "Evidence of viral adaptation to HLA class I-restricted immune pressure in chronic hepatitis C virus infection," Journal of Virology, vol. 80, no. 22, pp. 11094-11104, 2006.

[13] J. J. Lasarte, M. García-Granero, A. López et al., “Cellular immunity to hepatitis $\mathrm{C}$ virus core protein and the response to interferon in patients with chronic hepatitis C," Hepatology, vol. 28, no. 3, pp. 815-822, 1998.

[14] D. K. Wong, D. D. Dudley, P. B. Dohrenwend et al., "Detection of diverse hepatitis $\mathrm{C}$ virus (HCV)-specific cytotoxic T lymphocytes in peripheral blood of infected persons by screening for responses to all translated proteins of HCV," Journal of Virology, vol. 75, no. 3, pp. 1229-1235, 2001.

[15] A. Zabaleta, L. Arribillaga, D. Llopiz et al., "Induction of potent and long-lasting CD4 and CD8 T-cell responses against hepatitis $\mathrm{C}$ virus by immunization with viral antigens plus poly(I:C) and anti-CD40," Antiviral Research, vol. 74, no. 1, pp. 25-35, 2007.

[16] L. Arribillaga, A. L. D. De Cerio, P. Sarobe et al., "Vaccination with an adenoviral vector encoding hepatitis $\mathrm{C}$ virus (HCV) NS3 protein protects against infection with $\mathrm{HCV}$-recombinant vaccinia virus," Vaccine, vol. 21, no. 3-4, pp. 202-210, 2002.

[17] A. Folgori, S. Capone, L. Ruggeri et al., "A T-cell HCV vaccine eliciting effective immunity against heterologous virus challenge in chimpanzees," Nature Medicine, vol. 12, no. 2, pp. 190197, 2006.

[18] J. S. Zur Wiesch, G. M. Lauer, J. Timm et al., "Immunologic evidence for lack of heterologous protection following resolution of HCV in patients with non-genotype 1 infection," Blood, vol. 110, no. 5, pp. 1559-1569, 2007.

[19] H. Komatsu, G. Lauer, O. G. Pybus et al., "Do antiviral CD8+ T cells select hepatitis $\mathrm{C}$ virus escape mutants? Analysis in diverse epitopes targeted by human intrahepatic CD8+ T lymphocytes," Journal of Viral Hepatitis, vol. 13, no. 2, pp. 121-130, 2006.

[20] L. Uebelhoer, J. Han, B. Callendret et al., "Stable cytotoxic T cell escape mutation in hepatitis $\mathrm{C}$ virus is linked to maintenance of viral fitness," PLoS Pathogens, vol. 4, no. 9, Article ID e1000143, 2008.

[21] P. A. Reche, H. Zhang, J. Glutting, and E. L. Reinherz, "EPIMHC: a curated database of MHC-binding peptides for customized computational vaccinology," Bioinformatics, vol. 21, no. 9, pp. 2140-2141, 2005.

[22] B. Peters, J. Sidney, P. Bourne et al., "The immune epitope database and analysis resource: from vision to blueprint," PLoS Biology, vol. 3, no. 3, p. e91, 2005.
[23] R. C. Edgar, "MUSCLE: multiple sequence alignment with high accuracy and high throughput," Nucleic Acids Research, vol. 32, no. 5, pp. 1792-1797, 2004.

[24] C. E. Shannon, "The mathematical theory of communication," The Bell System Technical Journal, vol. 27, pp. 379-423; 623-656, 1948.

[25] C. M. Diez-Rivero and P. A. Reche, "CD8 T cell epitope distribution in viruses reveals patterns of protein biosynthesis," PLoS ONE, vol. 7, Article ID e43674, 2012.

[26] P. A. Reche, D. B. Keskin, R. E. Hussey, P. Ancuta, D. Gabuzda, and E. L. Reinherz, "Elicitation from virus-naive individuals of cytotoxic T lymphocytes directed against conserved HIV-1 epitopes," Medical Immunology, vol. 5, p. 1, 2006.

[27] M. Garcia-Boronat, C. M. Diez-Rivero, E. L. Reinherz, and P. A. Reche, "PVS: a web server for protein sequence variability analysis tuned to facilitate conserved epitope discovery," Nucleic Acids Research, vol. 36, pp. W35-W41, 2008.

[28] P. A. Reche and E. L. Reinherz, "Sequence variability analysis of human class I and class II MHC molecules: functional and structural correlates of amino acid polymorphisms," Journal of Molecular Biology, vol. 331, no. 3, pp. 623-641, 2003.

[29] J. J. Stewart, C. Y. Lee, S. Ibrahim et al., "A Shannon entropy analysis of immunoglobulin and T cell receptor," Molecular Immunology, vol. 34, no. 15, pp. 1067-1082, 1997.

[30] P. A. Reche, J.-P. Glutting, H. Zhang, and E. L. Reinherz, "Enhancement to the RANKPEP resource for the prediction of peptide binding to MHC molecules using profiles," Immunogenetics, vol. 56, no. 6, pp. 405-419, 2004.

[31] P. A. Reche, J. P. Glutting, and E. L. Reinherz, "Prediction of MHC class I binding peptides using profile motifs," Human Immunology, vol. 63, no. 9, pp. 701-709, 2002.

[32] P. A. Reche and E. L. Reinherz, "Prediction of peptide-MHC binding using profiles," Methods in Molecular Biology, vol. 409, pp. 185-200, 2007.

[33] K. Cao, J. Hollenbach, X. Shi, W. Shi, M. Chopek, and M. A. Fernández-Viña, "Analysis of the frequencies of HLA-A, B, and $\mathrm{C}$ alleles and haplotypes in the five major ethnic groups of the United States reveals high levels of diversity in these loci and contrasting distribution patterns in these populations," Human Immunology, vol. 62, no. 9, pp. 1009-1030, 2001.

[34] J. Robinson, J. A. Halliwell, H. McWilliam, R. Lopez, P. Parham, and S. G. Marsh, "The IMGT/HLA database," Nucleic Acids Research, vol. 41, pp. D1222-D1227, 2013.

[35] S. F. Altschul, T. L. Madden, A. A. Schäffer et al., "Gapped BLAST and PSI-BLAST: a new generation of protein database search programs," Nucleic Acids Research, vol. 25, no. 17, pp. 3389-3402, 1997.

[36] S. J. Hubbard and J. M. Thornton, NACCESS, Computer Program, Department of Biochemistry and Molecular Biology, University College London, London, UK, 1993.

[37] L. Swadling, P. Klenerman, and E. Barnes, "Ever closer to a prophylactic vaccine for HCV," Expert Opinion on Biological Therapy, vol. 13, no. 8, pp. 1109-1124, 2013.

[38] C. T. Nguyen and T. T. Tran, "Hepatitis vaccination and prophylaxis," Clinics in Liver Disease, vol. 13, no. 2, pp. 317-329, 2009.

[39] J. P. Davis, "Experience with hepatitis A and B vaccines," American Journal of Medicine, vol. 118, supplement 10, pp. 7S15S, 2005.

[40] H. G. Rammensee, J. Bachmann, N. P. N. Emmerich, O. A. Bachor, and S. Stevanović, "SYFPEITHI: database for MHC 
ligands and peptide motifs," Immunogenetics, vol. 50, no. 3-4, pp. 213-219, 1999.

[41] M. J. Blythe, I. A. Doytchinova, and D. R. Flower, "JenPep: a database of quantitative functional peptide data for immunology," Bioinformatics, vol. 18, no. 3, pp. 434-439, 2002.

[42] M. Bhasin, H. Singh, and G. P. S. Raghava, "MHCBN: a comprehensive database of MHC binding and non-binding peptides," Bioinformatics, vol. 19, no. 5, pp. 665-666, 2003.

[43] C. M. Diez-Rivero, M. Garcia-Boronat, and P. A. Reche, "Integrating T-cell epitope annotations with sequence and structural information using DAS," Bioinformation, vol. 3, no. 4, pp. 156$158,2008$.

[44] Q. Zhang, P. Wang, Y. Kim et al., "Immune epitope database analysis resource (IEDB-AR)," Nucleic Acids Research, vol. 36, pp. W513-W518, 2008.

[45] W. Zhong, P. A. Reche, C. Lai, B. Reinhold, and E. L. Reinherz, "Genome-wide characterization of a viral cytotoxic T lymphocyte epitope repertoire," Journal of Biological Chemistry, vol. 278, no. 46, pp. 45135-45144, 2003.

[46] M. Wang, K. Lamberth, M. Harndahl et al., "CTL epitopes for influenza A including the H5N1 bird flu; genome-, pathogen-, and HLA-wide screening," Vaccine, vol. 25, no. 15, pp. 28232831, 2007.

[47] R. Draenert, S. Le Gall, K. J. Pfafferott et al., "Immune selection for altered antigen processing leads to cytotoxic T lymphocyte escape in chronic HIV-1 infection," Journal of Experimental Medicine, vol. 199, no. 7, pp. 905-915, 2004.

[48] A. Sayle and E. J. Milner-White, "RASMOL: biomolecular graphics for all," Trends in Biochemical Sciences, vol. 20, no. 9, pp. 374-376, 1995.

[49] J. W. Yewdell and J. R. Bennink, "Immunodominance in major histocompatibility complex class I-restricted T lymphocyte responses," Annual Review of Immunology, vol. 17, pp. 51-88, 1999.

[50] P. A. Reche and E. L. Reinherz, "Definition of MHC supertypes through clustering of MHC peptide binding repertoires," in Artificial Immune Systems, pp. 189-196, Springer, Berling, UK, 2004.

[51] P. A. Reche and E. L. Reinherz, "PEPVAC: a web server for multi-epitope vaccine development based on the prediction of supertypic MHC ligands," Nucleic Acids Research, vol. 33, no. 2, pp. W138-W142, 2005.

[52] A. Sette and J. Sidney, "Nine major HLA class I supertypes account for the vast preponderance of HLA-A and -B polymorphism," Immunogenetics, vol. 50, no. 3-4, pp. 201-212, 1999.

[53] A. Sette and J. Fikes, "Epitope-based vaccines: an update on epitope identification, vaccine design and delivery," Current Opinion in Immunology, vol. 15, no. 4, pp. 461-470, 2003.

[54] H. H. Bui, J. Sidney, K. Dinh, S. Southwood, M. J. Newman, and A. Sette, "Predicting population coverage of T-cell epitopebased diagnostics and vaccines," BMC Bioinformatics, vol. 7, p. 153, 2006. 


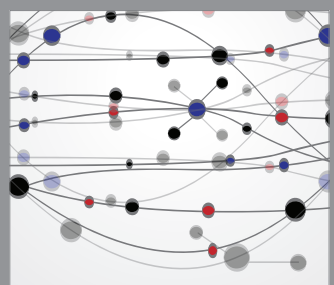

The Scientific World Journal
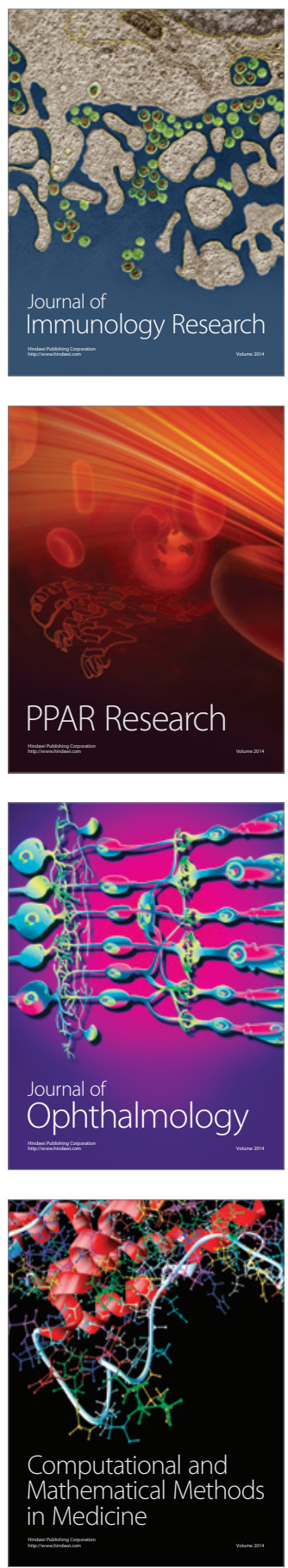

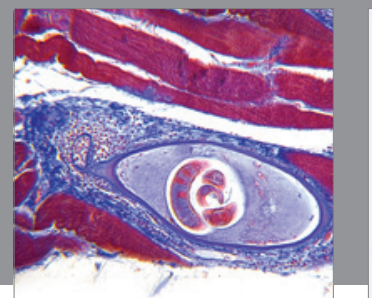

Gastroenterology

Research and Practice
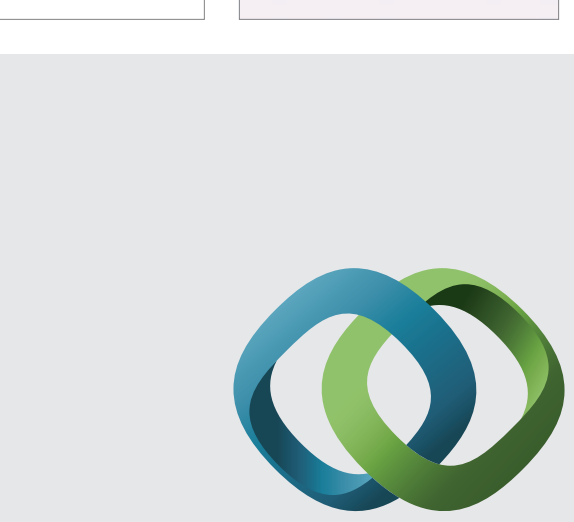

\section{Hindawi}

Submit your manuscripts at

http://www.hindawi.com
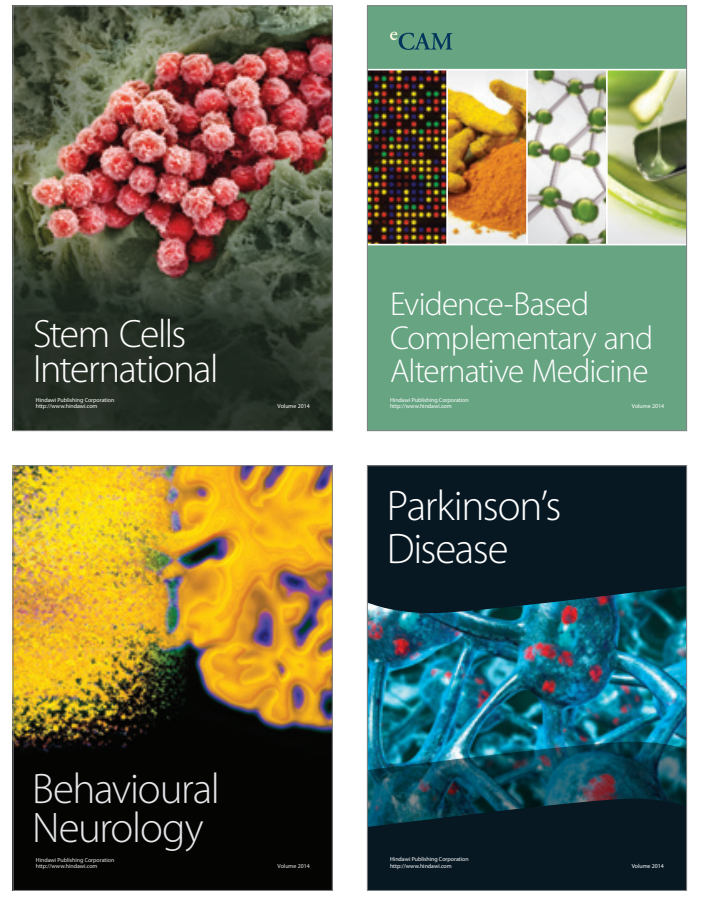
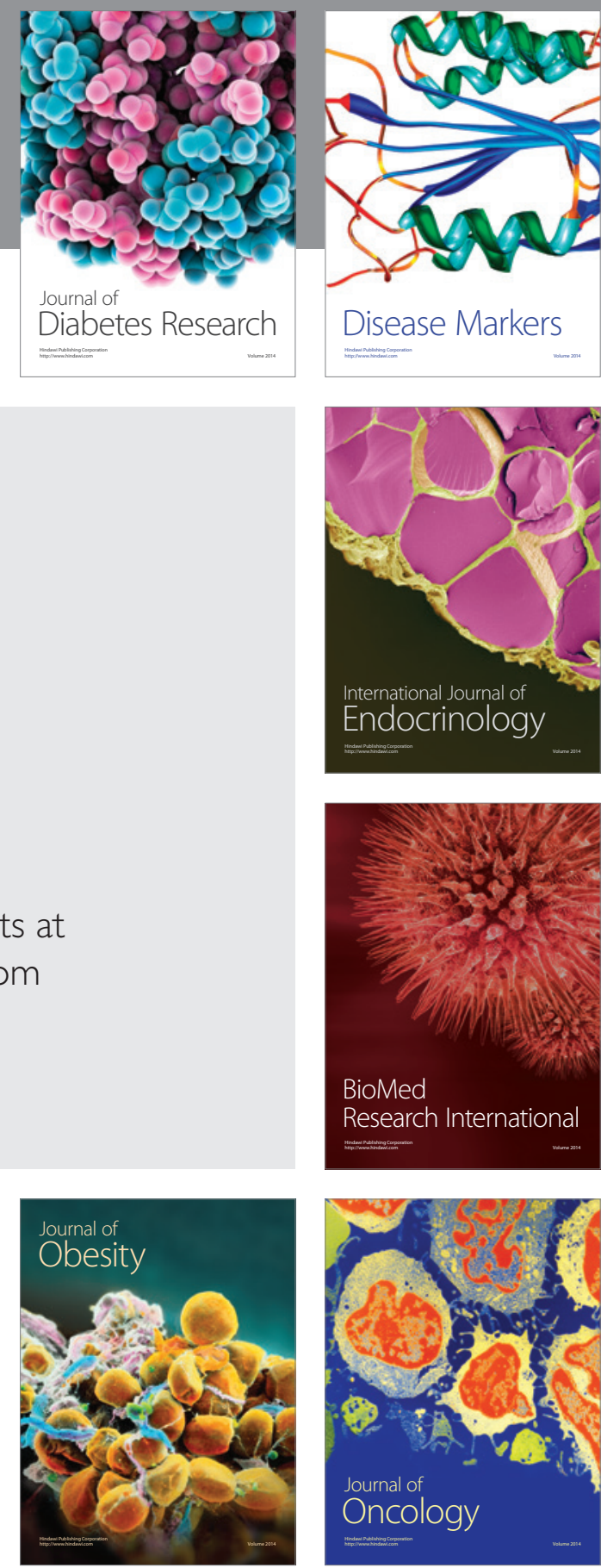

Disease Markers
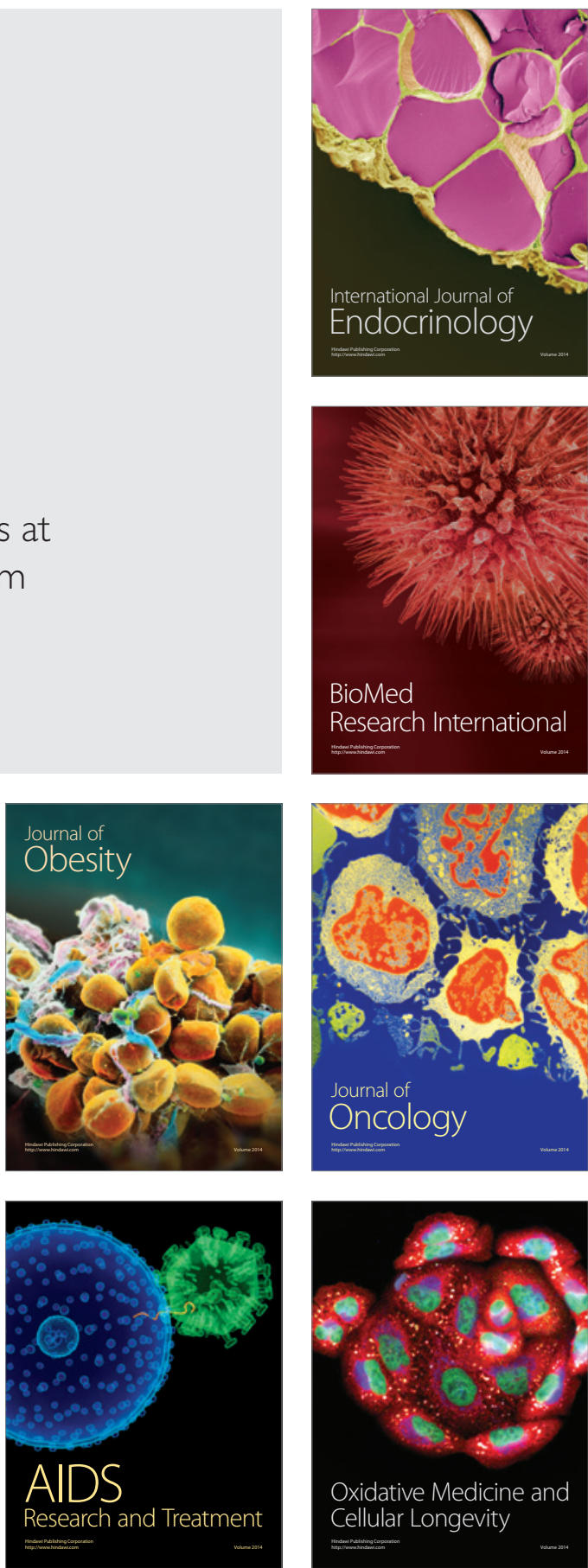